\title{
Time and Quantum Theory: A History and a Prospectus
}

\author{
Tom Pashby
}

September 26, 2013

$=$

\section{Introduction}

The conventional wisdom regarding the role of time in quantum theory is this: "time is a parameter in quantum mechanics and not an operator" (Duncan \& Janssen, 2013, p. 216, original emphasis). The reason for this is 'Pauli's theorem,' a collection of results that show that (subject to a mild restriction on the Hamiltonian) conventional quantum mechanics does not permit the definition of a time observable, i.e. a self-adjoint operator canonically conjugate to energy. ${ }^{1}$ If one wishes to have time appear as a genuine observable of the theory, then this is obviously a problem, called by some "the problem of time in quantum mechanics" (Hilgevoord \& Atkinson, 2011; Olkhovsky, 2011). Hilgevoord's (2005) attempted resolution of the problem rests on his rejection of a particular motivation that one might have for wishing to regard time as a genuine observable. Hilgevoord's argument is essentially this: there is nothing problematic about time being represented by a parameter rather than an operator since space is represented by a parameter rather than an operator as well.

In his otherwise excellent recent historical survey Hilgevoord (2005) contends that the demand that time be an observable can be traced back to a conceptual confusion common among the progenitors of quantum mechanics, in particular Dirac, Heisenberg, Schrödinger, and von Neumann. I will argue that the conceptual confusion is somewhat less severe, and the motivations somewhat more subtle, than Hilgevoord alleges. Hilgevoord claims that the expectation of the authors of quantum mechanics that time should be an

\footnotetext{
${ }^{1}$ See Srinivas \& Vijayalakshmi (1981) for a rigorous derivation of this result.
} 
observable was due to this confusion between space and position: led by the role of position as an observable of the theory, they were mistakenly led to the idea that time should be observable too. He traces the source of the confusion to the frequent use of the spatial co-ordinates $(x, y, z)$ to denote the spectral values of the position observable of a single particle $\left(q_{x}, q_{y}, q_{z}\right)$.

When presented with an operator whose spectral values appear to correspond to points of space, it is natural to expect also an operator whose spectral values correspond to instants of time. And given the expectation of these authors that quantum mechanics would ultimately be a relativistic theory, it is reasonable to demand of a theory set in space-time that time and space should appear on the same footing. However, as Hilgevoord points out, the spectral values of position are not identical with spatial pointsthis correspondence is only valid for a system comprising a single particle. In general the dimension of configuration space (and so the spectrum of the position observable) is $3 N$, where $N$ is the number of particles. Once this confusion is made apparent and it is realized that time $t$ (a parameter) is to be contrasted with space $(x, y, z)$ (also parameters) the apparent asymmetry is removed and so the justification for defining a time operator (i.e. a time observable) is removed, or so Hilgevoord claims. This leads him to dismiss later developments, such as the recent use of POVMs (Positive Operator Valued Measures) to define (generalized) time observables, as conceptually confused for the same reason (Hilgevoord \& Atkinson, 2011).

Now, with regard to this particular justification for regarding time as an operator, I agree that Hilgevoord offers an apt diagnosis: what is being contrasted here is not time and space, but time and position. But while I agree wholeheartedly that it would be a mistake to confuse space, time and position in this way, I am not convinced that this was a confusion to which many (or perhaps any) of the authors of quantum theory were prone. Indeed, Hilgevoord (2005) acknowledges that there were other reasons which led to the expectation that temporal properties were apt for representation by operators. It is my view that these other reasons for defining time operators were more important to those authors - I will claim that some remain compelling today — and thus are not so easily dismissed as resulting from a simple conceptual error.

In this paper I will be concerned with analyzing in more detail how ideas and expectations regarding the role of time in the theory arose and evolved in the early years of quantum mechanics, from 1925-27. The general theme will be that expectations which seemed reasonable from the point of view of matrix mechanics and Dirac's $q$-number formalism became implausible in light of Dirac-Jordan transformation theory, and were dashed by von Neu- 
mann's Hilbert space formalism which came to replace it. Nonetheless, I claim that the physical motivations behind these expectations were often sound, and in particular I will identify two concerns that remain relevant today. The first of these concerns Hilgevoord's accusation of conceptual confusion against those who seek a 'time observable.' The crux of my argument is the idea that time observables in quantum theory need not 'measure time' (as would a physical clock) but may instead serve to provide probability measures for the occurrence of events at particular (sets of) times, just as the position observable provides probability measures for the occurrence of events at particular (sets of) spatial points. This provides the means to resist Hilgevoord's accusation of pursuing a false analogy since if 'the' time observable concerns the location of an event in time, then it is no false contrast to draw an analogy with the position observable, which concerns the location of an event in space (the event in question being, at first blush, something like 'the particle's being here').

Secondly, I point out Dirac's use of an 'extended' classical phase space (which includes time and energy as conjugate variables from the outset) to define his quantum dynamics means that the corresponding quantum variables are not subject to Pauli's 'no-go' theorem (nor later related results) and, moreover, his motivations for using this extended configuration space are not merely relativistic. This indicates another way to avoid this 'problem of time:' by defining an 'extended' Schrödinger equation for functions of space and time we can have a quantum theory in which time and (minus the) energy are represented by canonically conjugate observables, as Dirac had originally intended. I will also attempt to show how these considerations are related: exploring the first claim (that the time of an event is an observable quantity) leads naturally to the second (that quantum theory should be defined on extended configuration space).

The structure of the paper is as follows. In Sections 2-4 I survey three historical episodes in early quantum theory that are relevant to my claims. Section 2 is concerned with matrix mechanics and the reasons behind attempts to define time as an observable (i.e. a matrix) in that context, and their later discrediting at the hands of Pauli. Section 3 tells the story of an ill-fated (and short lived) interpretation of matrix mechanics as a theory of 'quantum jumps,' events occurring at definite times. Section 4 contains a detailed exploration of the ways that time entered into Dirac's early quantum theory, arguing that his motivations for regarding time as an operator were quite distinct from those in the matrix mechanics camp, and thus untouched by Pauli's complaints. In Section 5 I make a positive argument for the consideration of event time observables as providing legitimate grounds 
for considering time operators and survey the impact of 'Pauli's theorem.' Section 6 contains some details of the history of attempts to define event time observables while avoiding these no-go results, ultimately as time shift covariant POVMs. I conclude in Section 6 by advocating a recent proposal by Brunetti et al. (2010) that links the use of event time observables to Dirac's extended Schrödinger equation.

\section{Time in Matrix Mechanics}

The expectation that energy and time must form a canonically conjugate pair arose within the matrix mechanics camp from the close relation of the new quantum mechanics to the action-angle form of classical (Hamiltonian) mechanics that inspired it. In Heisenberg's famous Umdeütung (Heisenberg, 1925) time plays an almost identical role in the description of the new quantum variables as it did in the specific classical cases he sought to re-interpret. The classical equation of motion, Hamilton's equation in action-angle co-ordinates $(J, w)$, read

$$
\frac{d w}{d t}=\frac{\partial H}{\partial J} \quad ; \quad \frac{d J}{d t}=-\frac{\partial H}{\partial w} .
$$

The time dependence in action-angle form is particularly simple since the canonical transformation into these co-ordinates is chosen such that $\dot{J}=0$ and $\dot{w}=v_{0}$, a constant. Thus $J$ is time independent and $w$ is linear in $t$. This being the case, a general solution $x(t)$ of these equations (for periodic systems) may be Fourier decomposed into a sum over components labeled by amplitude and phase:

$$
x(t)=\sum_{n=-\infty}^{\infty} \sum_{\tau \pm 1} A_{\tau}\left(J_{n}\right) e^{2 \pi i \tau v_{n} t},
$$

with the sum over $\tau= \pm 1$ serving to ensure that $x(t)$ is real. Thus it was this special form of classical Hamiltonian mechanics - one in which time dependence takes an especially simple form - that provided the basis of the emerging quantum kinematics. As we can see from the position of the variable $v_{n}$ in the above expression, the time evolution of these solutions was entirely confined to a set of complex phases, and so it was to be in the new quantum theory.

In the matrix mechanics of Born \& Jordan (1925) kinematical quantities are represented by Hermitian matrices whose time dependence takes the 
same form,

$$
\boldsymbol{p}(t)=p(n m) e^{2 \pi i v(n m) t} ; \boldsymbol{q}(t)=q(n m) e^{2 \pi i v(n m) t} .
$$

Having obtained a matrix representation of these kinematical quantities, it follows from the relation

$$
v(n m)=W_{n}-W_{m}
$$

that the time derivative of an arbitrary matrix function $\boldsymbol{g}(\boldsymbol{p q})$ may be written

$$
\dot{\boldsymbol{g}}=\frac{i}{\hbar}\left[\left(W_{n}-W_{m}\right) g(m n)\right]=\frac{i}{\hbar}(\boldsymbol{W} \boldsymbol{g}-\boldsymbol{g} \boldsymbol{W}),
$$

where $\boldsymbol{W}=\delta_{m n} W_{n}$ is a diagonal matrix (pp. 288-9). Since the diagonal form of $\boldsymbol{W}$ was critical to the validity of this relation, the major practical difficulty of applying the new quantum mechanics to a particular system with a classical Hamiltonian of known functional form became essentially that of finding a basis in which the quantum mechanical energy became a diagonal matrix.

By writing the Hamiltonian matrix $\boldsymbol{H}$ as a function of $\boldsymbol{p}$ and $\boldsymbol{q}$ Born and Jordan derived the following dynamical equations for quantum variables in the same form as Hamilton's equations in classical mechanics, ${ }^{2}$

$$
\left(\frac{\partial H}{\partial q}=\right) \dot{\boldsymbol{p}}=\frac{i}{\hbar}[\boldsymbol{H} \boldsymbol{p}-\boldsymbol{p H}] \quad ;\left(-\frac{\partial H}{\partial p}=\right) \dot{\boldsymbol{q}}=\frac{i}{\hbar}[\boldsymbol{H} \boldsymbol{q}-\boldsymbol{q H}] .
$$

They argued that the same relation holds for a general function $\boldsymbol{g}(\boldsymbol{p q})$ as well, yielding the so-called Heisenberg equation of motion,

$$
\dot{\boldsymbol{g}}=\frac{i}{\hbar}[\boldsymbol{H} \boldsymbol{g}-\boldsymbol{g} \boldsymbol{H}]
$$

which immediately gave the result that $\dot{\boldsymbol{H}}=0$, i.e. that energy is conserved. These results formed the basis of the more detailed analysis of the Dreimännerarbeit of Heisenberg, Born and Jordan (1925), where the problem of solving the equations of motion was reduced to the problem of finding a transformation $\boldsymbol{S}$ of canonical variables $\boldsymbol{q}_{0}, \boldsymbol{p}_{0}$ such that $\boldsymbol{S H}\left(\boldsymbol{q}_{0} \boldsymbol{p}_{0}\right) \boldsymbol{S}^{-1}$ is a diagonal matrix.

\footnotetext{
${ }^{2}$ See Van Der Waerden (1967, p. 49) for skepticism about the validity of the derivation.
} 


\subsection{Discussion and Postscript}

There is in this formalism no reason to suppose that time could not be represented by a matrix, and the fact that in classical mechanics the angle $w$ behaves very much like a time parameter is suggestive of the idea that there should be a matrix $\boldsymbol{t}(\boldsymbol{q p})$ canonically conjugate to $\boldsymbol{H}$ (which, as we've seen, is constant with time). Indeed, if one demands that this matrix $\boldsymbol{t}$ vary linearly with time then (2) appears to imply that it is canonically conjugate to energy $\boldsymbol{H}$ since

$$
\dot{\boldsymbol{t}}=1 \Rightarrow[\boldsymbol{H} \boldsymbol{t}-\boldsymbol{t} \boldsymbol{H}]=-i \hbar .
$$

The heuristic underlying this relation is quite different to Dirac's motivation for regarding time and energy as conjugates (which I turn to Section 4). Here, the time matrix $\boldsymbol{t}$ is defined as having a particular property, namely varying linearly with time (in analogy to the classical angle variable), and it is seen that this leads to the relation of conjugacy with $\boldsymbol{H}$. In contrast, Dirac kept the Hamiltonian conceptually and formally distinct from the operator conjugate to time, as evidenced by the fact that in Dirac's scheme it is not energy that is conjugate to time but minus the energy.

This distinction is important to bear in mind when considering Heisenberg's later analysis of the uncertainty equations where he appears to regard the canonical pair (energy, time) as equivalent to (action, angle) (Heisenberg, 1927b, p. 66). Specifically, Heisenberg makes use of the conjugacy relation (3), which suggests that he had this motivation in mind rather than Dirac's, in which case the sign is correct (contra Hilgevoord Hilgevoord (2005, p. 44)). However, as Hilgevoord reports (pp. 45-46), Heisenberg's analysis of the Stern-Gerlach experiment fails to elucidate this relation physically due to several unfortunate confusions (which I will pass over). Despite this confusion, Heisenberg's paper contains another physical interpretation of the time-energy uncertainty relation, which I address in the following section.

A more severe difficulty for the relation (3) was raised by Pauli (1933), following up on work by Schrödinger, who had considered the uncertainty relations for a 'quantum clock' embodying this canonical pair. Schrödinger pointed out that if such a system had definite value for the time variable then the value of its energy would be completely uncertain, a situation that he considered "physically meaningless" (Hilgevoord, 2005, pp. 49-50). Pauli (1933), on the other hand, argued that the equation above, which states that $\boldsymbol{H}$ and $\boldsymbol{t}$ are conjugate variables, implies that $\boldsymbol{t}$ is the generator of shifts in energy, just as position is the generator of shifts in momentum. Since the shift in energy was arbitrary this implied, he argued, that the existence of a Hermitian matrix $\boldsymbol{t}$ is inconsistent with the energy $\boldsymbol{H}$ having discrete 
eigenvalues, as in the case of a harmonic oscillator.

Later it was realized that this result only holds rigorously if the exponentiated Weyl form of the canonical commutation relations are assumed to hold for $H$ and $T$, which is a strictly stronger condition. ${ }^{3}$ In that case, the Stone-von Neumann theorem entails that every such canonical pair is unitarily equivalent to the Schrödinger representation of position and momentum, and thus every operator that features in such a pair has the spectrum of the real line (Prugovečki, 1971, p. 342). This conflicts with a different requirement on the spectrum of the Hamiltonian operator, namely that it be bounded from below. ${ }^{4}$

Evidently the Hamiltonian for the harmonic oscillator and free particle obey this condition since there is a state of lowest energy in those cases, but we might also want to impose the condition that a Hamiltonian have a spectrum bounded from below generally as a hallmark of physical reasonableness. This result provides the justification for the conventional wisdom that time in quantum mechanics is not an operator, and lies at the heart of the difficulties with the time-energy uncertainty relation - since time and energy are not a conjugate pair, the standard rigorous derivations of uncertainty relations do not apply. ${ }^{5}$

\section{The Time of a Quantum Jump}

Evidently, the question of whether time and energy are conjugate variables is closely related to the existence of a time-energy uncertainty principle. Reading Heisenberg's famous anschaulichen Inhalt paper today (Heisenberg, $1927 \mathrm{~b}$ ), one is struck by the centrality of the time-energy uncertainty relation in his informal discussions of the 'intuitive content' of the theory. It is clear that this relation is just as important as the position-momentum uncertainty relation for Hesienberg's attempt to articulate a physical interpretation of the theory. As he was to later put it: "I wanted to start from the fact that quantum mechanics as we then knew it [i.e. matrix mechanics] already

\footnotetext{
${ }^{3}$ Ironically, the number and phase operators of the harmonic oscillator do not form such a pair, so that Pauli's 'theorem' fails and one can construct a (periodic) time observable conjugate to the Hamiltonian. See Galindo (1984); Garrison \& Wong (1970) for a discussion of the distinction between the Weyl and Heisenberg forms and an explicit counterexample; see Galapon (2002) for a detailed critique of Pauli's original argument.

${ }^{4}$ See Section 2 of Srinivas \& Vijayalakshmi (1981) for a detailed analysis and several proofs of this result.

${ }^{5}$ For a recent discussion of such difficulties and attempts to surmount them see Busch (2007).
} 
imposed a unique physical interpretation" (Heisenberg \& Pomerans, 1971, p. 76).

Looking more closely, we see that Heisenberg's concern was often not with time in the abstract (i.e. on a par with space) but rather the relationship between the energy of the system and the time of a particular event-a so-called "quantum jump," regarded as a real physical process. Take the following passage:

"According to the physical interpretation of quantum theory aimed at here, the times of transitions or "quantum jumps" must be as concrete and determinable by measurement as, say, energies in stationary states. The spread within which such an instant is specifiable is $\ldots h / \Delta E$, if $\Delta E$ designates the change in energy in a quantum jump." (Heisenberg, 1927a, p. 77)

This illustrates his faith that the observable content of the theory should be fixed by theory, but also suggests the view was that these quantum jumps took place at definite moments of time, albeit times about which we have limited knowledge.

Moreover, Heisenberg discusses how the possibility of measuring a definite value of the energy depends on performing a measurement between the moments at which jumps occurred.

In quantum mechanics, this behavior [of interruption by quantum jumps] is to be interpreted in these terms: as the energy is really changed by external perturbations or quantum jumps, every energy measurement, insofar as it is to be unequivocal, must be performed in the interval between two disturbances. ${ }^{6}$ (Heisenberg, 1927a)

The implication is that a quantum system is to be understood as having a determinate energy at all times, but that this energy fluctuates due to exchanges with the environment or between subsystems - quantum jumps.

This was the view he had taken in his previous paper regarding energy exchanges between two coupled systems, which had inspired Dirac's transformation theory. ${ }^{7}$ Given this view, we come to appreciate why the timeenergy uncertainty relation has such a central role for the interpretation of the theory: since Heisenberg regarded the physical content of the theory as corresponding to discontinuous processes of energy exchange occurring at

\footnotetext{
${ }^{6} \mathrm{I}$ have made minor amendments to the translation here.

${ }^{7}$ See (Duncan \& Janssen, 2013, pp. 177-179) for more details.
} 
definite times, the energy-time relation was naturally of central importance to his project of providing an intuitive grasp of the physical content of the new mechanics.

It is also of interest that Jordan's view at this time is very similar to Heisenberg's, and one imagines that this is something that they had discussed together. In his Habilitation lecture (later published in English as a Nature article of 1927) Jordan chose to address the question of indeterminism with reference to the time of a single quantum jump:

What predictions can our theory make on this point? The most obvious answer is that the theory only gives averages, and can tell us, on the average, how many quantum jumps will occur in any interval of time. Thus, we must conclude, the theory gives the probability that a jump will occur at a given moment; and thus, so we might be led to conclude, the exact moment is indeterminate, and all we have is a probability for the jump. But this last conclusion does not necessarily follow from the preceding one: it is an additional hypothesis. (from Duncan \& Janssen 2013, pp. 187-188).

It seems that Jordan is taking here a very similar view to Heisenberg: the view that quantum jumps are physical events taking place at some definite time. In the first part of his answer, Jordan seems to approach Dirac's opinion that "[the theory] enables one to calculate the fraction of the total time during which the energy has any particular value, but it can give no information about the times of the transitions" (Dirac, 1927, p. 622). But Jordan goes further to say that there is information here about the rate of occurrence of quantum jumps. He goes even further in suggesting that the theory might be considered to supply information about the probability that a jump might occur at a particular time. This is distinguished from the view that the moment of time at which the jump occurs is indeterminate, leaving open the possibility that the probabilities involved could be interpreted objectively rather than epistemically. However, Jordan is not prepared to commit himself either way on this question.

What is clear is that there was at this time a strong tendency to regard quantum jumps (i.e. energy exchanges) as concrete physical processes occurring at determinate moments of time. In retrospect, this physical picture clearly had its roots in Bohr's atomic theory. By substituting the energy eigenstates of the new quantum mechanics for the stationary states of the old quantum theory one would naturally come to regard matrix mechanics as a theory of discontinuous transitions between energy eigenstates. This 
physical picture informed Heisenberg's initial discussion of the time-energy uncertainty relation, which arose in this context from considering the time of an event. This is a distinct role for time in quantum theory not considered by Hilgevoord, who treats time as an external co-ordinate to be measured by a physical clock.

\subsection{Postscript and Discussion}

The period of time during which this 'quantum jump' interpretation of the theory remained plausible was short-lived. By the time of Heisenberg's presentation with Born at the Solvay conference of October 1927 he no longer held the opinion that the time of a quantum jump was a physically meaningful quantity. Born and Heisenberg's presentation contains the following passage,

If one asks the question when a quantum jump occurs, the theory provides no answer. At first it seemed that there was a gap here that might be filled with further probing. But soon it became apparent that this is not so, rather, that it is a failure of principle, which is deeply anchored in the nature of the possibility of physical knowledge. One sees that quantum mechanics yields mean values correctly, but cannot predict the occurrence of an individual event. (Bacciagaluppi \& Valentini, 2009, p. 384)

This seems to represent a retreat to Dirac's position that only the time average was a physically meaningful quantity. However, the rest of their presentation reveals a more radical point of view. Bacciagaluppi and Valentini read their claim that "matrix mechanics deals only with closed periodic systems, and in these there are no changes. In order to have true processes ... one must restrict one's attention to a part of the system" (Bacciagaluppi \& Valentini, 2009, p. 185) to suggest that they shared the view of Campbell (endorsed by Heisenberg in a letter to Pauli) that time is a statistical phenomenon, absent in atomic systems but emerging at the macroscopic level like temperature or pressure.

Since the time-independent Schrödinger equation is solved by the stationary states corresponding to eigenfunctions of energy, if one makes the supposition that a system is always in such a state and the theory supplies probabilities for the 'jump' from one state to another, then it would be as if time did not exist except for these discontinuous transitions. Though Schrödinger introduces it only to reject it, his report contains a detailed analysis of this proposal, in which quantum systems considered as a whole 
involve no passage of time, and according to which time emerges from the theory as a macroscopic parameter related to the number of quantum jumps occurring between subsystems. According to this view there is no change, and thus no passage of time in between quantum jumps. Rather, time emerges as a parameter related to the rate at which jumps occur.

Limiting our attention to an isolated system, we would not perceive the passage of time in it any more than we can notice its possible progress in space. ... What we would notice would be merely a sequence of discontinuous transitions, so to speak a cinematic image, but without the possibility of comparing the time intervals between transitions. (Bacciagaluppi \& Valentini, 2009, p. 409)

According to Campbell's hypothesis, "one cannot regard the jump probability in the usual way as the probability of a transition calculated relative to unit time" (p. 409). On this view, the theory supplies probabilities for transitions between states and, in terms of temporal information, can only provide probabilities that one transition occur before or after another.

The alternative, says Schrödinger, is to regard the system not as occupying a single stationary state (along the lines of Bohr's earlier atomic theory) but rather as having a state that may be an arbitrary linear superposition of energy eigenstates. Taking this view-which was, of course, the view to win out - time appears in terms of the evolution of the relative phases of the energy eigenstates. This became the modern Schrödinger picture, in which the state but not the observables varies with time and the dynamics is given by the Schrödinger equation, which provides a alternative to the Heisenberg picture suggested by matrix mechanics (in which the observables vary according the the Heisenberg equations of motion rather than the state). There is more to say here about the emergence of the modern notion of state and of measurement, some of which is covered by Duncan \& Janssen (2013), but we can see that already these developments were fatal to the idea of the quantum jump as a discontinuous transition between stationary states, which relied critically on the hypothesis that a system remain in an energy eigenstate at all times.

The failure of the quantum jump interpretation, however, should not be read as prohibition on the introduction of the notion of the time of an event into quantum theory. The downfall of the idea of the time of a quantum jump, and ultimately the quantum jump itself, was not idea that the theory should supply probabilities for the temporal distribution of the occurrence of particular events, but the reliance on the idea that a system must exist 
in an eigenstate of energy. This idea, which can be traced directly back through the old quantum theory to Bohr's model of the hydrogen atom, was to prove an unjustified restriction on the development of quantum theory. Without the expansion of the theory to consider superpositions of energy eigenstates, there could be no time development of the system state (in the Schrödinger picture), and the state would be essentially frozen in time.

To those familiar with the so-called problem of time in canonical quantum gravity this situation will sound eerily familiar: there, the 'timeless' Wheeler-DeWitt equation seems to suggest that the dynamics of the universe (a closed system) are similarly frozen. ${ }^{8}$ Moreover, similar pronouncements about the elimination of time from physical theory abound in the interpretative literature, e.g. Barbour (1999); Earman (2002). If there is a lesson from history to be found here then perhaps it is this: the existence and nature of dynamics in quantum theory can be quite sensitive to background interpretative assumptions regarding the system state. Thus one might be advised to adopt a cautious attitude towards arguments that suggest the elimination of time from one's ontology on the basis of prototypical forms of quantum theories.

\section{Time as a $q$-number: Dirac's Classical Analogy}

Dirac, working in relative isolation in Cambridge, was led to the dynamical equations of matrix mechanics by pursuing a structurally richer classical analogy. Like Born and Jordan, he recognized the non-commutativity of the multiplicative operation as the key feature of Heisenberg's quantum variables, but, rather than focusing on a particular (matrix) representation of the variables, Dirac's approach led him to identify shared algebraic operations of the classical and quantum theories. Here we will briefly follow his development of the theory in his initial paper 'The Fundamental Equations of Quantum Mechanics' (Dirac, 1925).

Whereas Born and Jordan's derivative operation came for free from their use of matrix multiplication, Dirac sought to define his operation algebraically from the two basic conditions such an operation much satisfy: distributivity and the Leibniz law. He shows that the operation $a x-x a$, which is to say the commutator of two ' $q$-numbers' (meaning quantum numbers - a nomenclature introduced in a subsequent paper) satisfies these two conditions and so can be interpreted as a differentiation of $x$ with respect to some parameter $v$. For a special case, Dirac let $a$ be the diagonal matrix repre-

\footnotetext{
${ }^{8}$ This is briefly discussed by Bacciagaluppi \& Valentini (2009, p. 188).
} 
senting the energies of the allowed transitions, in which case $v$ is the time and the commutator returns $\dot{x}$, just as Born and Jordan had found.

But in contrast to Born and Jordan, who built up their dynamical equations from matrix operations acting according to the quantum condition, Dirac instead sought to establish a correspondence between classical and quantum operations by setting up a structural analogy between the two theories. He argued that as the quantum numbers become large the quantum commutator corresponds to the classical Poisson bracket (multiplied by a factor of $-i \hbar)$.

$$
\{x, y\}=\sum_{r}\left\{\frac{\partial x}{\partial q_{r}} \frac{\partial y}{\partial p_{r}}-\frac{\partial y}{\partial q_{r}} \frac{\partial x}{\partial p_{r}}\right\}
$$

The Poisson bracket is a canonical invariant, meaning that it takes the same value evaluated in any canonical co-ordinates. Moreover, the Poisson bracket expressions satisfy the two demands Dirac placed on an operation of differentiation. This suggested to him that the quantum commutator represented the same mathematical operation, valid for non-commuting ' $q$ numbers' - his own version of the correspondence principle.

Once this correspondence was established, the quantum equation of motion (2) followed immediately from the corresponding classical Poisson bracket by mere transcription according to Dirac's new quantum schema. The difference in his approach was manifest in his ability to import results from classical mechanics directly into his theory (although he was soon to see that his translation procedure led to ordering ambiguities). Since actionangle variables are classical conjugates with $\{w, J\}=1$ (having been reached by a canonical transformation) the suggestion is very strong indeed that $w$ and $J$, considered as $q$-numbers, must also be a canonical pair. Indeed, obtaining numerical results from Dirac's theory required transcription of the results of the corresponding classical problem, expressed in action-angle form. ${ }^{9}$

\section{1 'Relativity Quantum Mechanics'}

When Dirac came to consider relativistic quantum physics his approach was, naturally enough, to define a suitable relativistic classical description in terms of Poisson Brackets, and then apply his quantum translation prescription (Dirac, 1926b). ${ }^{10}$ It is worth quoting in full Dirac's description of

\footnotetext{
${ }^{9}$ See Darrigol (1993, pp. 309 -329) for a detailed discussion of this period.

${ }^{10}$ Note that this work was done before he was aware of Schrödinger's wave mechanics.
} 
this procedure and his view of the significance of defining suitable classical canonical variables, and his purposes for doing so.

It will be observed that the notion of canonical variables plays a very fundamental part in the theory. Any attempt to extend the domain of the present quantum mechanics must be preceded by the introduction of canonical variables into the corresponding classical theory, with a reformulation of the classical theory with P.B.'s [Poisson Brackets] instead of differential coefficients. The object of the present paper is to obtain in this way the extension of the quantum mechanics to systems for which the Hamiltonian involves the time explicitly and to relativity mechanics. (Dirac, 1926b, pp. 406-7, emphasis added)

Hilgevoord (2005, pp. 38-40) appears to take the view that it was the latter relativistic motivation that Dirac found most compelling, and that neither motivation was to survive the subsequent developments that led to Dirac's transformation theory (which survives today in the so-called Dirac-von Neumann formalism). I will argue instead that the former motivation, which doesn't not involve relativity, was important to Dirac's development of the theory, and does indeed persist within Dirac's thought, at least up until the genesis of transformation theory, recorded in Dirac (1927).

Returning to Dirac's paper of 1926, in the following section entitled "Quantum Time," Dirac immediately claims that "[t]he principle of relativity demands that the time shall be treated on the same footing as the other variables and so it must therefore be a $q$-number" (p. 407). In order to do so, Dirac defines a classical Poisson bracket that includes time $t$ as a variable along with its canonical conjugate $-W$ (i.e., minus the total energy).

$$
\{x, y\}=\sum_{r}\left\{\frac{\partial x}{\partial q_{r}} \frac{\partial y}{\partial p_{r}}-\frac{\partial y}{\partial q_{r}} \frac{\partial x}{\partial p_{r}}\right\}-\frac{\partial x}{\partial W} \frac{\partial y}{\partial t}+\frac{\partial y}{\partial W} \frac{\partial x}{\partial t}
$$

In defining this Poisson Bracket, the set of canonical variables is extended by two to include $t$ and $-W$, and so the dynamics of the system now takes place in this extended phase space. The physical solutions are defined by the demand that the Hamiltonian (defined on the extended phase space) vanishes with the total energy $W$, what is called today a constraint equation,

$$
H-W=0 .
$$

So while $t$ and $-W$ are variables conjugate on the extended phase space (leading to the quantum commutators detailed in Dirac's equation (7)) the 
dynamics of the system are confined to a subspace of the phase space defined by this constraint (called today the constraint surface). ${ }^{11}$

Note that there is nothing about the use of extended phase space which implies that the system in question is relativistic, since it is just the fact that the Poisson bracket is defined on the extended phase space which implies time and (minus the) energy are conjugate variables. Although considering a relativistic Hamiltonian provides a compelling motivation to consider the extended phase space, it is not the only motivation one might have, and there is no reason not to consider a non-relativistic system in this context. ${ }^{12}$ Hilgevoord (2005) regards the use of relativistic arguments to motivate the demand that energy and time be canonical conjugates in quantum mechanics as misguided due to the limited role that relativistic particle mechanics plays in classical (p. 36) and quantum physics (p. 42). Regardless, it remains the case that (classically) the conjugacy of energy and time has little to do with the fact that a system is relativistic, and everything to do with the fact that the system's dynamics are defined in the extended phase space. Although in the case of a relativistic system the use of extended phase space is unavoidable, it is clear that Dirac also viewed the (non-relativistic) Schrödinger equation as a wave equation defined on functions of space and time, i.e. as an equation that applies in the extended phase space.

\subsection{The Time-Dependent Schrödinger Equation}

Introduced by means of an optical-mechanical analogy, Schrödinger's wave mechanics was initially met with hostility from the matrix mechanics camp. However, as we have seen, Dirac's $q$-number approach was not tied to a matrix representation, and was as a result more flexible for extension in other directions. Following Heisenberg's discovery of a connection between the solutions of Schrödinger's wave equation and the energy eigenvalues that of the Hamiltonian matrix, Dirac seized on Schrödinger's new approach, which provided the means to free himself from overly restrictive reliance on classi-

\footnotetext{
${ }^{11}$ In fact, Dirac notes that the conjugacy relations may be inconsistent with the constraint, but this is just to say that the relations don't necessarily hold for functions on the constraint surface. Without the Schrödinger equation in hand, it is not yet clear that the dynamical equation is in fact a wave equation satisfied by certain functions, whereas the relations of conjuagacy hold generally for functions of the extended configuration space. It is also worth emphasizing that, whereas position and momentum are conjugate variables on both the unextended and extended phase space, energy and time are conjugate variables on the extended phase space alone.

${ }^{12}$ See, for example, Dirac's later development of this formalism (Dirac, 1966).
} 
cal methods. ${ }^{13}$ Unencumbered by the conceptual baggage of Schrödinger's painstaking path through classical physics, Dirac's starting point was the realization that quantum systems could be described by functions obeying a linear wave equation, and he quickly moved to explore the consequences.

In a remarkable paper 'On the Theory of Quantum Mechanics' (Dirac, 1926a) he laid out the essentials of an approach which would serve as the basis for the later integration of wave and matrix mechanics. He demonstrated the power of this new formalism by deriving the Bose-Einstein and Fermi-Dirac statistics for an assembly of identical systems from elementary conditions on the permutation of the wavefunctions describing the individual systems. The foundation of this approach was the recognition that Schrödinger's theory allowed for the explicit representation of conjugate variables as differential operators. To write down the time-dependent wave equation, therefore, merely required him to make the substitutions

$$
p_{r}=-i \hbar \frac{\partial}{\partial x} ; \quad-W=-i \hbar \frac{\partial}{\partial t}
$$

into the equation (4) above, treated as a wave equation, i.e.,

$$
(H-W) \psi=0 .
$$

Hence Dirac's derivation of the time-dependent equation depended on the extended phase space description described in (Dirac, 1926b). To explain: the replacement of the conjugate variable $-W$ by the corresponding differential operator relies on the existence of a space of functions of $t$ on which it acts. The implication is that $t$ is also a $q$-number, now an operator that acts by multiplication on this space of functions of extended configuration space. $^{14}$

In order to set up a correspondence with the Heisenberg equations of motion Dirac is required to fix the value of the ( $c$-number) variable $t$, but in doing so he makes it quite clear that the functions (or superpositions of functions) that satisfy the general wave equation are functions of time and space.

As an example of a constant of integration of the dynamical system take the value $x\left(t_{0}\right)$ that an arbitrary function $x$ of the $p$ 's and $q$ 's, $W$ and $t$ has at a specified time $t=t_{0}$. The matrix

\footnotetext{
${ }^{13}$ See (Darrigol, 1993, pp. 329-333) for a discussion of the difficulties introduced by the degeneracy of energy levels arising in systems of many particles.

${ }^{14}$ Note again that the space of functions on which $W$ acts is not identical with the space of wavefunctions that satisfy the wave equation (5).
} 
that represents $x\left(t_{0}\right)$ will consist of elements each of which is a function of $t_{0}$. (Dirac, 1926a, p. 665)

Under the special condition that the Hamiltonian is time independent (i.e. a constant of integration), so that the energy $W$ has a diagonal matrix representation, Dirac was able to derive the time dependence of the matrix elements of a $q$-number $x$ (although, as he is at pains to point out, only for a Hamiltonian that does not involve time explicitly). This reverses the logical order of Born and Jordan's derivation of (1), which assumed the time dependence by means of Heisenberg's classical analogy. Dirac here instead shows how this time dependence arises from the dynamics of quantum mechanics, that is, the Schrödinger equation.

However, Dirac explicitly states that he views this separation of time and space as inessential, and describes the alternative (solving directly in terms of the extended phase space without considering variation in $t$ ) as more fundamental:

It should be noticed that the choice of the time $t$ as the variable that occurs in the elements of the matrices representing variable quantities is quite arbitrary, and any function of $t$ and the $q$ 's that increases steadily would do. ... It is probable that the representation of a constant of integration of the system by a matrix of constant elements is more fundamental than the representation by a matrix whose elements are functions of some variable such as $t \ldots$ (Dirac, 1926a, p. 666)

In summary, we can see that there was another motivation, independent from relativistic considerations, which led Dirac to regard energy and time as conjugate variables: his expression for the time-dependent Schrödinger equation required that energy be represented by a differential operator $-i \hbar d / d t$, which was defined on a space of functions of time. Note also that Dirac did not yet have a dynamics that could apply to time-dependent Hamiltonians, since the treatment he had given assumed from the outset that the allowed energies were time independent. He remained convinced that this would be found by considering the Hamiltonian dynamics in extended phase space.

\subsection{Time in Dirac's Transformation Theory}

The culmination of Dirac's work on the foundations of quantum mechanics was his transformation theory, which aimed to give a unified framework for both wave and matrix mechanics. In Dirac's presentation of transformation 
theory, 'The Physical Interpretation of the Quantum Dynamics' (1927) it is apparently assumed from the outset that the theory involves the split between space and time that had been introduced in the earlier paper.

These matrix elements [of a dynamical variable $g$ ] are functions of the time only. In the present paper we shall not take relativity mechanics into account, and shall count the time variable wherever it occurs as merely a parameter (a $c$-number). (Dirac, 1927 , p. 625)

However, although time is not explicitly considered by Dirac as an operator, I will argue that there remains a role for the extended phase space in conceptualizing time development within the theory, and in particular the inclusion of time-varying Hamiltonians, which Dirac had originally announced as a important motivation for using the extended phase space.

In the course of the development of the transformation theory, which Dirac intends to provide a generalization of matrix mechanics to address non-periodic systems and continuous observables, he makes the (oft-quoted) crucial link with Schrödinger's wave mechanics:

The eigenfunctions of Schrödinger's wave equation are just the transformation functions ... that enable one to transform from the (q) scheme of matrix representation to a scheme in which the Hamiltonian is a diagonal matrix (Dirac, 1927, p. 635, original emphasis)

As Heisenberg had proposed, these eigenfunctions are in fact the energy eigenstates, so what Dirac has found at this stage is the connection to Schrödinger's time-independent wave equation, which appears in the following form,

$$
H\left(q_{r}^{\prime},-i \hbar \frac{\partial}{\partial q_{r}^{\prime}}\right)\left(q_{r}^{\prime} / \alpha^{\prime}\right)=H\left(\alpha^{\prime}\right)\left(q_{r}^{\prime} / \alpha^{\prime}\right) .
$$

It remains for him to provide a link to the time-dependent equation that he had derived previously, i.e. the Heisenberg equations of motion. Remarkably, he does not do so: in his presentation of the theory the time dependence of the quantum variables is apparently assumed from the outset (condition (ii) of p. 327, ibid.). Neither does he try to derive the time-dependent Schrödinger equation in a way that we would recognize today. In fact, although the time-dependent Schrödinger equation does make an appearance in the paper, it does so almost by accident, as the conclusion to an argument that seeks to establish the dynamics for a time-varying Hamiltonian. Indeed, 
the only place Dirac explicitly considers time dependence of the solutions of the Schrödinger equation is in considering time varying Hamiltonians: first in general (p. 635) and then as a perturbation (p. 640). I contend that this indicates that, in 1927, Dirac viewed that state as a time-independent object, unless acted on in a time-varying way.

In the first part of the paper Dirac had only identified his transformations with energy eigenstates, and then relied on the relation to the extended classical phase space to consider dynamics. The time variation of the quantum variables he first considered - "constants of integration" - was particularly simple on this pseudo-classical picture. So long as the Hamiltonian was constant with time, the time variation was given by the Heisenberg equations of motion (2). However, if the Hamiltonian is time-dependent then the matrix scheme cannot have this simple time dependence. Dirac explains the problem as follows:

For systems in which the Hamiltonian involves the time explicitly, there will be in general no matrix scheme with respect to which $H$ is a diagonal matrix, since there will be no set of constants of integration that do not involve the time explicitly. (Dirac, 1927, p. 635)

Yet the result of the derivation that follows is an equation for a Hamiltonian $H$ that does not explicitly involve time - an equation that we immediately recognize today as the time-dependent Schrödinger equation for a timevarying wavefunction $\psi(x)$,

$$
H\left(q_{r},-i \hbar \frac{\partial}{\partial q_{r}}\right)\left(q^{\prime} / \alpha^{\prime}\right)=H\left(q_{r}, p_{r}\right)\left(q^{\prime} \alpha^{\prime}\right)=i \hbar \frac{\partial}{\partial t}\left(q^{\prime} / \alpha^{\prime}\right) .
$$

This equation (numbered (12) in Dirac's paper) is immediately followed by Dirac's claim that it gives "Schrödinger's wave equation for Hamiltonians that involve time explicitly" (p. 636). In discussing this equation, (Darrigol, 1993, p. 341) presents an alternative derivation (not Dirac's) which follows Dirac's earlier paper 1926a in assuming that $H$ is a constant of the motion. However, it is quite clear that this was not Dirac's intention, since he explicitly assumes that the Hamiltonian in question varies with time.

Dirac's initial comment about the lack of a matrix scheme with respect to which a time varying Hamiltonian is diagonal could be read in two ways: he could mean (a) that one and the same scheme will not suffice at every instant or (b) that the time dependent Hamiltonian cannot be brought into diagonal form at all. First note that Dirac in his treatment of time dependent 
perturbations (p. 640) shows that such a Hamiltonian can be written as a diagonal matrix whose entries depend on $t$. Thus he must be considering a time dependence here that cannot be written perturbatively. Dirac describes the properties of a matrix scheme on (p. 627). Dirac's condition (ii) gives the dynamics of the matrix scheme in terms of the Heisenberg equations of motion (2). Condition (iii) states that the Hamiltonian must be given as a diagonal matrix, but Dirac later says (on p. 628) that he will consider more general transformations for which that is not the case. This appears to refer to transformations which (e.g.) bring the matrices into the position basis, in which the Hamiltonian is not diagonal. This would be the case for any variable that does not commute with $H$, and Dirac makes it clear that he is considering here variables that do not have time dependence.

But in the derivation of the equation of motion for a time varying Hamiltonian, it is explicitly assumed that we are dealing with such time varying quantities, which thus moves us beyond the ambit of the Heisenberg equations of motion. Dirac begins the derivation (p. 635) by considering the Hamiltonian at an instant $t=\tau$, and the corresponding instantaneous variables $q_{\tau}, p_{\tau}$. Functions of $q_{\tau}$ and $p_{\tau}$ do not involve time explicitly, and so we can regard the Heisenberg equation of motion (for variation in $\tau$ ) as acting on these functions. From this it follows that

$$
H_{\tau}\left(q_{\tau}^{\prime} \alpha^{\prime}\right)=i \hbar \frac{\partial\left(q_{\tau}^{\prime} / \alpha^{\prime}\right)}{\partial \tau} .
$$

This first part of Dirac's derivation establishes that we can use a timevarying version of the Heisenberg equations of motion. But key to this move is the idea that $\tau$ is something like a gauge fixing parameter rather than a variable on which the constants of integration might depend. This indicates, I contend, that he begins by considering solutions of the 'extended' Schrödinger equation that lie in the extended configuration space, for which there are no eigenfunctions in the 'instantaneous' space of spatial functions.

However, Dirac immediately suggests (p. 636) that we write $t$ for $\tau$ and $q$ for $q_{\tau}$, which removes the time dependence of $H_{\tau}$ and gives (7). Hence does Dirac apparently arrive at the time-dependent equation for a time independent Hamiltonian. Now it is quite plausible that Dirac has merely suppressed the time dependence here on both sides, rather than dropping it entirely, so that we indeed have here the Schrödinger equation for timedependent Hamiltonians. ${ }^{15}$ Yet there is something odd about interpreting equation (7) in this way, since when applied to a time-independent

\footnotetext{
${ }^{15}$ Thanks to Prof. Fleming for pushing me on this point, which I here concede.
} 
Hamiltonian the right hand side would be zero, as there is no time variation in that case. What this shows is that Dirac was apparently unaware that the state could vary with time while the Hamiltonian did not, and was thus deeply wedded to (what we would call today) the Heisenberg picture. As Dirac interpreted it, then, this was not yet the modern day timedependent Schrödinger equation, which applies to both time-varying and time-independent Hamiltonians.

But it is clear in retrospect that equation (7) can be interpreted in precisely this way: comparing (6) with (7), we see that what Dirac had found was that the variation of the state $\left(q^{\prime} / \alpha^{\prime}\right)$ in time is given by the Hamiltonian operator in the position representation (rather than the energy representation). That Dirac apparently did not immediately recognize the equation in this way illustrates the pull of the extended phase space on his conception of the quantum state. As Dirac was aware, the space of instantaneous solutions was the only one in which his transformations could be defined. But in the course of the derivation, these instantaneous spaces were reached by fixing a particular value of the variable $t$ in the extended space and considering variation with respect to a parameterization $\tau$ of that value. That is, the time variation was now to be given in an instantaneous form. Thus did Dirac successfully, although haltingly, put an end to his earlier conception of the Schrödinger equation as involving an operator $-W$ conjugate to time.

The alternative was to consider a full blown four-dimensional wave equation applying to functions of time and space, a much more formidable problem. The middle ground that Dirac had found by dealing with the problem in this manner was to lead to the modern conception of the time-dependent Schrödinger equation, in which variation in time is given with respect to the parameterization of the unitary representation group of time translations, generated by $H$. But at this time- before he became aware of Hilbert space methods - Dirac did not possess the modern notion of a quantum state as a vector state. Moreover, the notion of a (Schrödinger picture) instantaneous state was one to which he was to remain resistant: specifying a time parameterization for the variation of the state meant that the state could not be given a relativistically invariant meaning. ${ }^{16}$

\footnotetext{
${ }^{16}$ The modern notion of state only appeared in the second edition of Dirac's Principles of Quantum Mechanics. See (Brown, 2006, p. 402-403) for more details. Also note that, contrary to Hilgevoord's claim that "this view disappeared from his later work" (Hilgevoord, 2005, pp. 36-37), the use of the extended phase space was essential to Dirac's work on constrained Hamiltonian mechanics, e.g. Dirac (1966).
} 


\subsection{Postscript and Discussion}

In the subsequent years the rise of von Neumann's Hilbert space formalism led to the grounding of the success of the Dirac-Jordan transformation theory in the results of functional analysis. Crucial to this development was von Neumann's proof of the spectral theorem in 1929, which allowed for the representation of unbounded self-adjoint operators like position by an integral over a spectral family of projection operators. ${ }^{17}$ Subsequently, Stone's theorem of 1930 established a role for self-adjoint operators as the generators of one-parameter unitary groups, which correspond to symmetry transformations (Prugovečki, 1971, pp. 331-338). This gives rise to an interpretation of the Weyl commutation relations as expressing a symmetrical relationship between two self-adjoint operators, i.e. that one is the generator of shifts in the spectrum of the other, and vice versa. As was discussed in Section 2.1, this framework is demonstrably incompatible with the introduction of a time observable, understood to be a self-adjoint operator canonically conjugate to the Hamiltonian.

Hence it is clear that, at least with respect to the current Dirac-von Neumann formalism, several of the expectations described in the previous section regarding the role of time in quantum theory turned out to be wrong: time and energy are not conjugate variables, the Schrödinger equation must be defined for functions of space alone, and there is no such thing as the time of a quantum jump (or, alternatively, of collapse). ${ }^{18}$ Nonetheless, I have shown that the motivations of the authors were not just the result of simple conceptual confusion, i.e., of mistaking one thing for another (although later physicists may have been misled along those lines). Instead, I have attempted to paint a more complex picture, revealing the distinctive motivations that led to two separate proposals for the conjugacy of time and energy.

To rephrase the two conjugacy relations we have arrived in the language of von Neumann's formalism, the relation of Heisenberg from Section 2 reads

$$
[H, T] \psi=-i \hbar \psi \text { for all } \psi \in \mathcal{H}
$$

(or at least a dense domain) and asserts the conjugacy of the Hamiltonian $H$ and a putative time observable $T$. As discussed in Section 2.1, the arguments of Pauli (1933) and those who followed serve to rule out the existence of such

\footnotetext{
${ }^{17}$ See (Duncan \& Janssen, 2013, pp. 240-241) for a discussion.

${ }^{18}$ Of course, the dynamics of the theory can be modified $\grave{a}$ la GRW to bring about a stochastic collapse process at particular moments of time, but I will not consider here these alternative dynamics.
} 
an operator. However, the relationship assumed by Dirac may be expressed

$$
\left[-W, T_{+}\right] \psi=-i \hbar \psi \text { for all } \psi \in \mathcal{H}_{+},
$$

where the similarity of form obscures the important point that the Hilbert space on which the operators $-W$ and $T_{+}$are to be defined is not the space on which $H$ is defined, i.e. $\mathcal{H} \neq \mathcal{H}_{+}$. To take a concrete example, let $\mathcal{H}=L^{2}\left[\mathbb{R}^{3}\right]$, which is apt for describing a single spinless particle. The instantaneous state of the system is thus given by a vector $\psi(x) \in \mathcal{H}$ (assuming the state is pure), where $x$ ranges over space. In contrast, $\mathcal{H}_{+}=L^{2}\left[\mathbb{R}^{4}\right]$, the space of square integrable functions on the extended configuration space, and vectors in this space $\psi(x, t) \in \mathcal{H}_{+}$are (superpositions of) functions of space and time.

Restrictions on the spectrum of $H$ are motivated by the fact that the eigenvalues of $H$ reflect the allowed energies of the system, but there is no analogous physical interpretation of $W$. Hence there is no restriction on the spectrum of $W$, and so (9) may harmlessly assert that $-W$ is the generator of shifts in time $T_{+}$, and vice versa. Thus the classically conjugate variables $t$ and $-W$ may be promoted to quantum operators on $\mathcal{H}_{+}$without running into the difficulties described in Section 2.1. With this understanding, there is no conceptual reason standing in the way of following Dirac's (1926) suggestion in the context of modern quantum theory, so long as it is understood that the formalism is to be extended to include functions of space and time. However, it remains to be shown that there a good motivation to do so, and a coherent interpretation of the theory that results. The rest of the paper is devoted to that task.

\section{In Defense of Event Time Observables}

Let us return to Hilgevoord's (2005) rejoinder that time already has an appropriate representation in the theory as a parameter, and so the use of extended phase space is misguided. In order to understand (and sidestep) these complaints it will be helpful to outline Hilgevoord's classification of the ways that time may arise in a physical theory (his Section 1.1). The main distinction that Hilgevoord draws is between external time and internal times. External (or co-ordinate) time $t$ is the partner of the spatial co-ordinates $x, y, z$ in a co-ordinatization of the space-time in which the system is situated, $(x, y, z, t)$. Hilgevoord contends that this role for external time suggests that it should remain a parameter rather than a variable, 
and regards the use of the extended phase space in classical mechanics as 'unnatural' (Hilgevoord, 2005, p. 36).

Internal times, by contrast, are to be found among

... the internal spatial and temporal variables connected with the specific physical systems the theory aims to describe, such as the position variables of particles and time variables of clocks. These variables are dynamical: they obey equations of motion. ... if we look for time operators we must look for internal times provided by special physical systems, 'clocks.' (Hilgevoord, 2005, p. 31)

Thus, for Hilgevoord, the only valid motivation for defining time operators comes from the canonical quantization of variables that describe physical clocks. However, this ignores a large body of foundational work in quantum theory regarding what I will term event time observables.

For example, in a recent review of the time-energy uncertainty relation Busch (2007) describes another role for time in addition to external and internal (or intrinsic) time, which he calls observable time. He motivates this definition as follows:

the study of dynamics often involves experimental questions about the time of an event, the time difference between events, or the duration of a process associated with the object system. This [also] raises the quest for a treatment of time as an observable. (Busch, 2007, p. 76)

Note that this motivation is quite distinct from the idea that external time should be promoted to a variable (for whatever reason). In this case, we begin with an experimental quantity - the time of an event, such as the moment that a Geiger counter clicks, or the duration of a process, such as tunneling time - and demand that the theory provide a suitable prediction for the distribution of these events in time (or durations). This motivation is, therefore, most closely related to Heisenberg and Jordan's notion of the time of a quantum jump, regarded as an event occurring at a definite moment of time (discussed in Section 3). However, there is an important difference since proponents of event time observables do not purport to be measuring the time of a quantum jump, or collapse, or what have you. The idea is that the events in question correspond to determinable outcomes of an experiment, which are considered to be distributed in time rather than, say, space.

In this case, then, the analogy to position is a good one: an event time observable concerns the position of an event in time just as the position 
observable concerns the position of an event in space. In this connection it may help to visualize a standard diffraction experiment, in which the experimentally determinable positions concern the location of a dot on, say, a photoluminescent screen. In this context, the event time observable concerns the distribution of times at which a dot appears, whereas the position observable concerns the distribution of spatial locations at which a dot appears. In modern quantum theory, to derive such a distribution from the system state we are required to define a suitable operator, customarily a selfadjoint operator. Observables are often defined by their properties under symmetry transformations, for example Jauch (1968, pp. 197-199) regards the covariance of the position operator with spatial translations (generated by the total momentum) as expressing the homogeneity of space. Essentially, Wightman (1962) shows that requiring this covariance is sufficient to uniquely define a self-adjoint position operator.

An event time observable is to be defined, therefore by its behavior under temporal translations, generated by the Hamiltonian. In particular, we will require such observables to covary with time translations. ${ }^{19}$ In effect, what this means is that the experimental results (depending on relative times) will be independent of the time at which the experiment begins, and so this expresses the homogeneity of time. However, 'Pauli's theorem' (Srinivas \& Vijayalakshmi, 1981, §2) entails that there is no time translation covariant Projection Valued Measure (PVM) mapping intervals of time to orthogonal projections, and, since the PVMs and self-adjoint operators are in one-toone correspondence, there is thus no self-adjoint operator corresponding to the time of an event. ${ }^{20}$ Nonetheless, there are time translation covariant Positive Operator Valued Measures (POVMs) which map intervals of time to positive operators with spectrum $[0,1] .{ }^{21}$

The first thing to note is that in the usual identification of observables with self-adjoint operators it is assumed that these these operators act instantaneously. In the Schrödinger picture (in which the states vary with time) the expectation value of an observable $A$ in the (pure) state $\psi_{t}=U_{t} \psi$ is $\langle A\rangle_{t}=\left\langle\psi_{t} \mid A \psi_{t}\right\rangle$, whereas in the Heisenberg picture (in which the observ-

\footnotetext{
${ }^{19}$ This is to be regarded as a necessary, not a sufficient condition.

${ }^{20}$ For a proof of this fact about PVMs see, e.g., (Teschl, 2009, Theorem 3.7, p. 97).

${ }^{21}$ An operator is positive, $A \geq O$, if $\langle\phi \mid A \phi\rangle \geq 0$ for all $\phi \in \mathcal{H}$. A (normalized) POVM is a map $X \mapsto E(X)$ from $X \in \mathcal{X}$, a $\sigma$-algebra of a nonempty set $\Omega$, to $E(X)$, a positive operator, such that: (i) $E(X) \geq O$; (ii) $E(\Omega)=\mathbb{I}$; (iii) $E\left(\cup X_{i}\right)=\sum E\left(X_{i}\right)$ for disjoint sequences $X_{i} \subset \mathcal{X}$. A PVM is a POVM such that $E(X)^{2}=E(X)$, or equivalently $E(X \cup Y)=E(X) E(Y)$, in which case I write $P(X)$. See Busch et al. (1995) for more details of their use in quantum theory.
} 
ables vary with time) the expectation value of an observable $A(t)$ is given by $\langle A(t)\rangle=\left\langle\psi\left|U_{t}^{\dagger} A U_{t}\right| \psi\right\rangle{ }^{22}$ In the Schrödinger picture it makes very little sense to ask when a particular event occurs (in the sense of a probability for it occurring during some interval of time) since we may only interrogate the state at a instant of time. In contrast,

"event time measurements are extended in time, with sensitive detectors waiting to be triggered. The experimenter has no control over the time instant at which the detectors fire. This very instant constitutes the outcome of such a measurement.(Busch, 2007, p. 19)

However, in the Heisenberg picture we may define operators that involve more than one instant of time (indeed, a measurable subset of instants) by integrating over $t$.

To take a simple example, consider an experiment consisting of a single radioactive atom and a Geiger counter that fully surrounds it. If the halflife of the atom is 1 hour, then the probability that the counter clicks in the first hour is $1 / 2$, the probability it clicks in the second hour $1 / 4$, the probability it clicks in the third hour is $1 / 8$, and so on. Thus the probability that the counter clicks at some point in the future is given by an arithmetic series that tends to one as $t$ tends to infinity. However, once the counter has been observed to click, the probability that it clicks in the future is essentially zero. This is evidently a experimentally meaningful situation, and we should expect that this phenomenological law may be derived from a detailed quantum mechanical description of the decay process. However, within the standard account of measurement there is provably no way to implement this simple scheme.

This description would involve a (Heisenberg picture) quantum mechanical vector state $\psi$ in a Hilbert space $\mathcal{H}$, a Hamiltonian $H$ describing the time evolution of the system, and a series of projection operators $T_{i}$ representing the proposition "the system decays during hour $i$ " such that $\left\langle\psi \mid T_{1} \psi\right\rangle=1 / 2$, $\left\langle\psi \mid T_{2} \psi\right\rangle=1 / 4$, and $\left\langle\psi \mid T_{3} \psi\right\rangle=1 / 8$, and so on. Requiring that the distribution of results respect the time translation symmetry implemented by the unitary group $U_{t}=e^{-i H t}$, we have that $T_{2}=U_{t}^{\dagger} T_{1} U_{t}, T_{3}=U_{t}^{\dagger} T_{2} U_{t}$, and so on (where $t$ is one hour). Even this bare bones sketch is enough to tell us something interesting about the operators $T_{i}$ : if $H$ is a self-adjoint operator with spectrum bounded from below then it follows that $\left\langle\psi\left|T_{i+1} T_{i}\right| \psi\right\rangle \neq 0$

\footnotetext{
${ }^{22}$ Evidently these return the same expectation value, $\langle A\rangle_{t}=\langle A(t)\rangle$, but they are not identical expressions.
} 
and so these operators $T_{i}$ cannot be projections onto mutually orthogonal subspaces of $\mathcal{H} .^{23}$

Thus there is no mixed state decomposition in terms of distinct eigenstates $\psi_{i}$ of $T_{i}$ (for which $T_{i} \psi_{i}=\psi_{i}$ ) such that the $\psi_{i}$ would correspond to the system decaying during distinct intervals of time, and neither can the $T_{i}$ together serve to define a self-adjoint 'time of decay' operator. The former implication indicates that von Neumann's famous projection postulate cannot be applied to this situation; the latter than the identification of observables of the theory with self-adjoint operators is ill-suited to include the time of an event as an observable quantity. Yet there seems every reason to suppose that the theory should be able to answer questions like, "When will the Geiger counter click?" or in a diffraction experiment, "When will a dot appear on the screen?" In failing to answer these questions, the theory would be fail to be empirically adequate; this failure would constitute a real 'problem of time' for the theory. But this problem can be overcome, and without modifying the dynamics: at first blush, the problem is not with the way that quantum mechanics defines the state of the system, but the way that probabilities are derived from the state.

\section{Defining Event Time Observables}

The history of attempts to define event time observables in quantum mechanics begins with Aharonov \& Bohm's (1961) critique of the energy-time uncertainty relation, understood as a limitation on the possibility of measuring the energy of a system precisely in an arbitrarily short time. In essence, Aharonov \& Bohm's objection amounts to the observation that the Hamiltonian is a self-adjoint operator like any other, and so can (according to the usual Dirac-von Neumann formalism) can be measured effectively at an instant. To combat what they see as a prevalence of somewhat heuristic arguments based on particular experimental setups adduced in support of the erroneous interpretation, they provide a detailed analysis of an experiment which serves as a counterexample, i.e. one in which the energy of the system under observation can be determined precisely in arbitrary short time. The 'apparatus' in this experiment is modeled by a free particle in one dimension, considered as providing a physical clock to measure the time at which the measurement interaction occurs.

\footnotetext{
${ }^{23}$ For a proof of this result see Unruh \& Wald (1989, p. 2606), whose argument is easily adapted to the current case if it is assumed that there is some $k>0$ such that for all $i>k$ the probability of decay is zero.
} 
However, the operator they suggest is closely related to the time of arrival of a classical free particle, and so it is plausible that the operator they define is better understood as representing the time of an event rather than a clock. The classical time of arrival is arrived at by a simple rearrangement of the expression for position as a function of time, $q(t)=q(0)+v t$. That is, the classical time of arrival at the point $x=0$ is

$$
t(q(0), p)=-q(0) \frac{m}{p} .
$$

Now, we immediately see that there is a problem with this definition when $p=0$, but this has a physical interpretation: a free particle with zero momentum will never arrive at the origin. ${ }^{24}$ Thus, in the classical case, it seems clear that $t(q(0), p)$ provides the time co-ordinate of a particular event: the event of the particle's passing the origin.

The first difficulty one encounters in transcribing this classical expression into quantum form is that $Q(0)=Q$ (the Schrödinger picture position operator) does not commute with $P$ (the momentum operator), so this expression contains an ordering ambiguity. Following the usual procedure of symmetrization (first suggested by Dirac) we obtain: ${ }^{25}$

$$
T_{a}=-\frac{m}{2}\left(Q P^{-1}+P^{-1} Q\right) .
$$

This operator displays a similar difficulty with $p=0$, which results in its domain of definition being restricted to functions (considered in the momentum representation) which behave suitably as $p \rightarrow 0$. This restriction entails that $T_{a}$, while symmetric on its domain, cannot be extended to a self-adjoint operator on $\mathcal{H}=L^{2}[\mathbb{R}]$. It is thus a maximally symmetric operator. ${ }^{26}$ Nonetheless, $T_{a}$ is canonically conjugate to $H=(1 / 2 m) P^{2}$ in the sense that (formally at least)

$$
\left[T_{a}, H\right]=\frac{1}{2}\left(P^{-1}\left[Q, P^{2}\right]+\left[Q, P^{2}\right] P^{-1}\right)=-i \hbar .
$$

\footnotetext{
${ }^{24}$ If $q(0) \neq 0$ then it will never get to the origin, if $q(0)=0$ then it will never leave and hence never arrive.

${ }^{25}$ This differs from the expression given by Aharonov \& Bohm (1961, p. 1652) by an overall sign. This presumably justifies their interpretation of the operator as a clock since the corresponding classical expression returns $q(t)=v t$, which entails that $q(0)=0$ so that the position of the particle is proportional to the elapsed time. Note that in that case the relevant position operator is the Heisenberg picture $Q(t)$ rather than $Q(0)=Q$.

${ }^{26}$ See (Egusquiza \& Muga, 1999, §3) for a recent discussion of these difficulties.
} 
But since $T_{a}$ is not a self-adjoint operator, this blocks the derivation of a Weyl commutation relation with $H$ and so $T_{a}$ avoids 'Pauli's theorem' of Section 2.1. ${ }^{27}$

This is a good illustration, then, of the mutually supportive relationship between the requirement that observables must correspond to self-adjoint operators and the idea that time covariance is constitutive of an event time observable: to the extent that event time observables are operators that covary with time shifts, $T_{a}$ is an event time observable, but to the extent that observables must be self-adjoint $T_{a}$ is not an observable at all. As I have suggested, the way out of this impasse is to allow for the experimentally observable quantity (the time of an event such as a detector firing) to be represented by a time covariant POVM. But this move does not involve the promotion of $T_{a}$ to an observable; the relation of $T_{a}$ to the corresponding time covariant POVM is more subtle, although there is a sense in which a maximally symmetric operator does uniquely determine such a POVM.

The first steps towards this understanding of the quantum time of arrival were taken by Allcock (1969) and Kijowski (1974). ${ }^{28}$ Kijowski took an axiomatic approach to defining the quantum distribution of arrival times. The axioms he chose were based on axioms that were sufficient to derive the classical distribution. One of these axioms ensured its characteristic covariance under time translations. Corresponding to the corresponding quantum distribution he found our time of arrival operator $T_{a}$. Since every maximally symmetric operator defines a unique POVM (Akheizer \& Glazman, 1993, Theorem 2, p. 135), this distribution can also be obtained from the POVM $I \mapsto E^{T_{a}}(I)$ so defined.

Allcock, on the other hand, provided the first analysis of the difficulties of the time of arrival in terms of the positivity of $H$. By means of an analytic continuity argument he was able to show that the condition $H \geq O$ entails the non-orthogonality of the (generalized) eigenstates of $T_{a}$, characteristic of its failure to be self-adjoint. ${ }^{29}$ He found that he could avoid this problem by 'doubling' the domain of definition of $T_{a}$ to include negative eigenstates of $H$ (i.e. negative energies), arriving at a new operator $T_{a}^{ \pm}$that was self-adjoint,

\footnotetext{
${ }^{27}$ Recall that Pauli's theorem rests on the fact that the Weyl commutation relations are logically equivalent to the mutual covariance of those operators with shifts generated by the other. But $T_{a}$ is not a self-adjoint operator and thus, given the one-to-one correspondence of self-adjoint operators and PVMs, there can be no PVM corresponding to $T_{a}$ at all. Therefore, it follows that $H$ does not covary with shifts in energy since $T_{a}$ generates no unitary group.

${ }^{28}$ For details of these and other interesting episodes in the development of the quantum time of arrival see Muga \& Leavens (2000).

${ }^{29}$ See, again, (Akheizer \& Glazman, 1993, Theorem 2, p. 135).
} 
but defined on a 'larger' Hilbert space $\mathcal{H}^{\prime} \supset \mathcal{H}$. This operator is canonically conjugate to the doubled Hamiltonian $H^{ \pm}$and, so $I \mapsto P^{T_{a}^{ \pm}}(I)$ is time shift covariant PVM on $\mathcal{H}^{\prime}$.

This provides another way to avoid Pauli's theorem, since the spectrum of $H^{ \pm}$is not bounded from below. However, the self-adjoint operator $T_{a}^{ \pm}$he defined (and its corresponding PVM) is of negligible physical relevance since the Hamiltonian of a free particle evidently does have a spectrum bounded from below. Nonetheless, as is well-known (although apparently not by Allcock), by projecting the operators $P^{T_{a}^{ \pm}}(I)$ to the original Hilbert space via an orthogonal projection $P_{\mathcal{H}}$ one obtains positive operators $E^{T_{a}}(I)=$ $P_{\mathcal{H}} P^{T_{a}^{ \pm}}(I)$, i.e. the positive operators that form the time of arrival POVM $I \mapsto E^{T_{a}}(I)$ defined by $T_{a}$. This result is an instance of Naimark's famous dilation theorem, which establishes that a POVM can always be extended to a PVM in a 'larger' Hilbert space in this way, to which it is related by a projection. ${ }^{30}$

Allcock and Kijowski can perhaps be forgiven for failing to making these connections, writing as they were at a time before the development of the POVM formalism by Holevo (1982), Kraus (1983), Ludwig (1983) and others (in response to various problems in quantum measurement theory). It was left to Werner (1986) to make the connection of the time of arrival to time covariant POVMs, which he did in some generality to arrive at a treatment of what he termed screen observables. Making use of results from operator algebra and Mackey's theory of imprimitivity, Werner obtained a general recipe for constructing POVMs covariant under a unitary group by means of their Naimark dilation. Screen observables are defined as POVMs covariant under the translation group of an arbitrarily chosen hyperplane. As such, they include the usual position observable (corresponding to an instantaneous hyperplane) and the time of arrival POVM just discussed (which, in three dimensions, corresponds to a timelike hyperplane). Werner showed that the resulting POVM could be uniquely defined (subject to some other restrictions) for both non-relativistic and relativistic symmetry groups.

Screen observables apply to a typical diffraction experiment where an electron, say, is emitted and some time later detected at a photoluminescent screen. Since the screen is sensitive to the presence of an electron at all times, and electrons in an ensemble will be detected at different times, an instantaneous position observable cannot suffice to describe even the spatial distribution of detection events. For these screen observables, one assumes

\footnotetext{
${ }^{30}$ See Egusquiza \& Muga (1999) for a complete discussion of this particular case, and (Busch et al., 1995, p. 31) for a statement of the theorem in this form.
} 
that the detector will fire at some time $t$, and so the sum of the probability of detection over all times is unity. Note that very few real experimental arrangements (if any) correspond to anything like an instantaneous position measurement, which (under this interpretation as a screen observable) would provide probabilities for a detector spread out through all of space which fires exactly once when switched on for an instant.

More recently, Brunetti \& Fredenhagen (2002) supply a general recipe for constructing event time observables from an instantaneous projection or positive operator (an effect) taken to correspond to the occurrence of an event. Key to this construction is the technique of operator normalization, by which a time covariant POVM is normalized according to the condition that the event in question occurs exactly once (in analogy with screen observables). A straightforward example concerns the detection of a particle within a detector occupying a volume of space $\Delta$ rather than a plane. In this case, the detection event is represented by the instantaneous projection $P_{\Delta}(t)=U_{t}^{\dagger} P_{\Delta} U_{t}$, with the probability of detection at a time $t$ for a system in the (Heisenberg picture) state $\psi$ given by $\left\langle\psi \mid P_{\Delta}(t) \psi\right\rangle$, .

Naively, one might expect the following positive operator to describe detection during the time interval $I=\left[t_{1}, t_{2}\right]$,

$$
T_{\Delta}(I):=\int_{t_{1}}^{t_{2}} P_{\Delta}(t) d t .
$$

However, there is no guarantee that the expectation value of $T_{\Delta}$ will be less than one, and so this is not a normalized POVM. Brunetti \& Fredenhagen's suggestion is to use the maximal such operator, in this case

$$
T_{\Delta}(\mathbb{R})=\int_{-\infty}^{\infty} P_{\Delta}(t) d t
$$

known as the dwell time operator, ${ }^{31}$ to provide a suitable normalization in the following way.

Being positive, $T_{\Delta}(\mathbb{R})$ has a unique positive square root such that $\left(T_{\Delta}(\mathbb{R})^{1 / 2}\right)^{2}=$ $T_{\Delta}(\mathbb{R})$. Using the inverse of this operator, we define

$$
E_{\Delta}(I)=T_{\Delta}(\mathbb{R})^{-1 / 2} T_{\Delta}(I) T_{\Delta}(\mathbb{R})^{-1 / 2} .
$$

It is easily verified that $E_{\Delta}(I)$ is a time translation covariant POVM. ${ }^{32}$ This operator normalized POVM returns $E_{\Delta}(\mathbb{R})=\mathbb{I}$ and so the probability

\footnotetext{
${ }^{31}$ See Jose Munoz et al. (2010) for a recent discussion.

${ }^{32}$ Brunetti \& Fredenhagen (2002) supply a general recipe for obtaining such a POVM from any instantaneous effect which takes considerably more care with domains of definition and so on.
} 
distribution obtained reflects the condition that the event given by $P_{\Delta}$ occurs at some $t$, i.e. this POVM supplies a conditional probability. ${ }^{33}$

Clearly, then, recent years have seen considerable progress made in the quest to define event time observables for quantum theory. The main puzzle raised by event time observables such as these is, in my view, one of providing a suitable update rule. The first worry I have is conceptual, the second technical. First, the very normalization of a POVM such as $E_{\Delta}(I)$ which made it suitable for its role as an event time observable makes it ill-suited to provide probabilities for events that occur subsequent to detection. For example, it is unclear that we can obtain a definite answer to the question: What is the probability that the particle is first detected in $\Delta$ during $I$ and subsequently in $\Delta^{\prime}$ ? To answer this question appears to require a new normalization scheme since now the particle is detected in both $\Delta$ and $\Delta^{\prime}$, i.e. twice. But if we normalize along those lines then we have lost the conditional nature of the probabilities desired: the question was, what is the probability of finding the particle in $\Delta^{\prime}$ given that it was already detected in $\Delta$ ?

Secondly, while POVMs play the same predictive role as PVMs,${ }^{34}$ POVMs are particularly ill-suited to supply the means to update the state, which is typically defined for a projection through Lüders' Rule, which projects the state into the eigenspace of the measured eigenvalue (normalizing according to the trace). That is, for a projection $P_{k}$ onto the eigenspace associated with $k$,

$$
\rho \rightarrow \tilde{\rho}=\frac{P_{k} \rho P_{k}}{\operatorname{tr}\left[P_{k} \rho\right]}
$$

This rule leads to the following expression for conditional probability

$$
\operatorname{Pr}(F \mid E)=\frac{\operatorname{tr}[E F E \rho]}{\operatorname{tr}[E \rho]}=\operatorname{tr}[F \tilde{\rho}]
$$

valid for projections $E, F$ such that $F \leq E$, where $\leq$ is the relation of subspace inclusion.

The fact that a positive operator $B \geq O$ has a unique square root $\left(B^{1 / 2}\right)^{2}=B$ leads to the standard generalization of Lüders' rule to posi-

\footnotetext{
${ }^{33}$ In connection with the time of arrival, Hoge (2008) verifies that the POVM $E^{T_{a}}(I)$ is returned from $E_{\Delta}(I)$ in the limit $|\Delta| \rightarrow 0$, that is, as detection within a volume becomes detection at a plane (or point).

${ }^{34}$ As with a projection, a normalized positive operator $E$ supplies probabilities through taking the trace of the density operator $\rho, \operatorname{Tr}[\rho E]$.
} 
tive operators (or effects), the so-called Lüders operation ${ }^{35}$

$$
\rho \rightarrow \bar{\rho}=\frac{B^{1 / 2} \rho B^{1 / 2}}{\operatorname{tr}[B \rho]},
$$

which reduces to Lüders' rule when $B^{2}=B$. By following the earlier example of (10) we would be led to define

$$
W(A \mid B)=\operatorname{tr}[A \bar{\rho}]=\frac{\operatorname{tr}\left[B^{1 / 2} A B^{1 / 2} \rho\right]}{\operatorname{tr}[B \rho]}
$$

as a conditional probability for the effect $A$ given the effect $B$. But it is easily seen that this does not have the form of a conditional probability (unless $B$ is a projection) since if $B^{2} \neq B$ then $W(B \mid B) \neq 1$.

This suggests that event time POVMs would be ill-suited to describe, say, a cloud chamber experiment in which a series of ionization events occur within the detector, one after another. However, Brunetti et al. (2010) provide the hint of a solution, which also provides a novel motivation for adopting Dirac's extended phase space. In this context, this amounts to consideration of a Hilbert space of functions of space and time $\psi(x, t) \in L^{2}\left[\mathcal{R}^{4}\right]=\mathcal{H}_{+}$ on which a self-adjoint time operator may be defined, the spectral projections of which provide the means to define event time observables as time covariant PVMs rather than POVMs. They would thus appear to be appropriate for the application of Lüders' rule, if only the trace were defined on this space.

The solution to this difficulty lies in considering instead the extended Schrödinger equation, defined for functions of time and space as Dirac originally envisioned it,

$$
(H-W) \psi(x, t)=0 .
$$

The problem with this equation is that the operator $(H-W)$ has a continuous spectrum, and so there is no vector $\psi(x, t) \in \mathcal{H}_{+}$which is an eigenvector with eigenvalue 0. Without going into the details, Brunetti et al. (2010) show how solutions to this equation may be written in terms of a linear functional rather than a vector. While these physical solutions don't form a Hilbert space, and define non-normalizable 'weights' on the algebra of observables of $\mathcal{H}_{+}$rather than algebraic states, there is a construction which, given an operator on $\mathcal{H}_{+}$representing the occurrence of an event, leads to a (GNS) Hilbert space representation giving the expectation values for (a subset of)

\footnotetext{
${ }^{35}$ See (Busch et al., 1995, p. 37).
} 
the algebra of observables on $\mathcal{H}_{+}$- the algebra of events - on the condition that the event in question did occur.

This weight thus provides a replacement for the trace, such that by conditionalizing on these events one can calculate probabilities for subsequent events, e.g. a detector's firing again elsewhere. This theory has a good claim to be regarded as a straightforward generalization of the usual Schrödinger dynamics, but the form that the theory takes is surprisingly distinct: the solutions of the extended Schrödinger equation do not form a Hilbert space, the dynamics are not given by a unitary operator, and there is no meaning to the phrase 'the state of the system' without first specifying an event (the occurrence of which can be used to give a probability assignment to further events). Such probability assignments thus appear to correspond to a conditional state. The interpretative possibilities offered by this 'extended' quantum theory are, I submit, exciting and deserve further exploration.

\section{References}

Aharonov, Y. \& Bohm, D. (1961). Time in the quantum theory and the uncertainty relation for time and energy. Physical Review, 122(5), 16491658 .

Akheizer, N. I. \& Glazman, I. M. (1993). Theory of Linear Operators in Hilbert Space, Vol. 2. New York: Dover.

Allcock, G. (1969). The time of arrival in quantum mechanics I: Formal considerations. Annals of Physics, 53(2), 253-285.

Bacciagaluppi, G. \& Valentini, A. (2009). Quantum theory at the crossroads: reconsidering the 1927 Solvay conference. Cambridge: Cambridge University Press.

Barbour, J. (1999). The End of Time. New York: Oxford University Press.

Born, M. \& Jordan, P. (1925). Über die streuung von strahlung durch atome. In van der Waerden (Ed.), Sources of quantum mechanics (pp. 223-252). Dover.

Brown, L. (2006). Paul A. M. Diracs the principles of quantum mechanics. Physics in Perspective (PIP), 8(4), 381-407.

Brunetti, R. \& Fredenhagen, K. (2002). Time of occurrence observable in quantum mechanics. Physical Review A, 66(4), 044101. 
Brunetti, R., Fredenhagen, K., \& Hoge, M. (2010). Time in quantum physics: from an external parameter to an intrinsic observable. Foundations of Physics, 40(9), 1368-1378.

Busch, P. (2007). The time-energy uncertainty relation. In G. Muga, A. Ruschhaupt, \& A. del Campo (Eds.), Time In Quantum Mechanics - Vol. 1 (2nd. Edition) (pp. 73-105). Springer.

Busch, P., Grabowski, M., \& Lahti, P. J. (1995). Operational quantum physics. Springer Verlag.

Darrigol, O. (1993). From c-numbers to q-numbers: the classical analogy in the history of quantum theory, volume 10. University of California Press.

Dirac, P. A. M. (1925). The fundamental equations of quantum mechanics. Proceedings of the Royal Society of London. Series A, 109(752), 642-653.

Dirac, P. A. M. (1926a). On the theory of quantum mechanics. Proceedings of the Royal Society of London. Series A, Containing Papers of a Mathematical and Physical Character, 100, 661-677.

Dirac, P. A. M. (1926b). Relativity quantum mechanics with an application to compton scattering. Proceedings of the Royal Society of London. Series A, 111(758), 405-423.

Dirac, P. A. M. (1927). The physical interpretation of the quantum dynamics. Proceedings of the Royal Society of London. Series A, 113(765), 621-641.

Dirac, P. A. M. (1966). Lectures on quantum mechanics. Belfer Graduate School of Science, Yeshiva University.

Duncan, A. \& Janssen, M. (2013). (never) mind your p's and q's: Von neumann versus jordan on the foundations of quantum theory. The European Physical Journal H, 38(2), 175-259.

Earman, J. (2002). Thoroughly modern McTaggart or what McTaggart would have said if he had read the general theory of relativity. Philosophers' Imprint, 2(3), 1-28.

Egusquiza, I. \& Muga, J. (1999). Free-motion time-of-arrival operator and probability distribution. Physical Review A, 61, 012104. 
Galapon, E. (2002). Pauli's theorem and quantum canonical pairs: the consistency of a bounded, self-adjoint time operator canonically conjugate to a Hamiltonian with non-empty point spectrum. Proceedings of the Royal Society of London A, 458(2018), 451-472.

Galindo, A. (1984). Phase and number. Letters in Mathematical Physics, $8(6), 495-500$.

Garrison, J. C. \& Wong, J. (1970). Canonically conjugate pairs, uncertainty relations, and phase operators. Journal of Mathematical Physics, $11,2242-50$.

Heisenberg, W. (1925). Über quantentheoretische umdeutung kinematischer und mechanischer beziehungen. In Van Der Waerden (1967), (pp. 261276).

Heisenberg, W. (1927a). The actual content of quantum theoretical kinematics and mechanics. Zhurnal Physik, 43, 172-198.

Heisenberg, W. (1927b). Über den anschaulichen Inhalt der quantentheoretischen Kinematik und Mechanik. Zeitschrift für Physik, 43, 172-198. (English translation in: Wheeler, J. A. and Zurek, W. H. (1983). Quantum theory and measurement. Princeton: Princeton University Press).

Heisenberg, W. \& Pomerans, A. (1971). Physics and beyond: Encounters and conversations. Harper \& Row New York.

Hilgevoord, J. (2005). Time in quantum mechanics: a story of confusion. Studies In History and Philosophy of Science Part B: Studies In History and Philosophy of Modern Physics, 36(1), 29-60.

Hilgevoord, J. \& Atkinson, D. (2011). Time in quantum mechanics. In The Oxford Handbook of Philosophy of Time. Oxford University Press.

Hoge, M. O. (2008). Relationale zeit in der quantenphysik. Master's thesis, University of Hamburg.

Holevo, A. (1982). Probabilistic and Statistical Aspects of Quantum Theory. Amsterdam: North-Holland.

Jauch, J. (1968). Foundations of quantum mechanics. New York: AddisonWesley. 
Jose Munoz, I. L. E., del Campo, A., Seidel, D., \& Muga, J. G. (2010). Dwelltime distributions in quantum mechanics. Time in Quantum Mechanics, 2,97 .

Kijowski, J. (1974). On the time operator in quantum mechanics and the heisenberg uncertainty relation for energy and time. Reports on Mathematical Physics, 6(3), 361-386.

Kraus, K. (1983). States, Effects and Operations: Fundamental Notions of Quantum Theory. Springer Verlag.

Ludwig, G. (1983). Foundations of Quantum Mechanics. Springer-Verlag.

Muga, J. G. \& Leavens, C. R. (2000). Arrival time in quantum mechanics. Physics Reports, 338(4), 353-438.

Olkhovsky, V. S. (2011). On time as a quantum observable canonically conjugate to energy. Physics-Uspekhi, 54, 839.

Pauli, W. (1933). Handbuch der Physik, chapter Die Allgemeine Prinzipien der Wellenmechanik, (pp. 83-272). Number 24. Berlin:Springer.

Prugovečki, E. (1971). Quantum mechanics in Hilbert space. Academic Press.

Srinivas, M. \& Vijayalakshmi, R. (1981). The 'time of occurrence' in quantum mechanics. Pramana, 16(3), 173-199.

Teschl, G. (2009). Mathematical methods in quantum mechanics: with applications to Schrödinger operators. Providence: American Mathematical Society.

Unruh, W. G. \& Wald, R. M. (1989). Time and the interpretation of canonical quantum gravity. Physical Review D, 40(8), 2598.

Van Der Waerden, B. L. (1967). Sources of Quantum Mechanics. Amsterdam: North-Holland.

Werner, R. (1986). Screen observables in relativistic and nonrelativistic quantum mechanics. Journal of mathematical physics, 27, 793.

Wightman, A. S. (1962). On the localizability of quantum mechanical systems. Reviews of Modern Physics, 34, 845-872. 